\title{
Study of Oligohydramnios Cases by Modified Biophysical Profile \& Their Perinatal Outcome
}

\author{
Dr. Somalatha Raparthy ${ }^{1}$, Dr. Channareddy Sunitha ${ }^{2}$ \\ ${ }^{1,2}$ (Sr. Resident, Asst. Prof in Department of OBGYN, Dr. PSIMS \& RF, Chinoutpalli, Krishna Dt, Andhra \\ Pradesh)
}

\begin{abstract}
:
Background:Assessment of foetal wellbeing is important, in timely diagnosis of foetalcompromise and management. Oligohydramnios is associated with increasedincidence of adverse perinatal outcomes like foetal distress, meconium stained liquor, lowAPGAR score, low birth weight, NICU admission, perinatal morbidity and mortality.Foetal biophysical profile is a well-established method of antepartumsurveillance. Classical biophysical profile needs two phase testing by ultrasound and external doppler monitor to record foetal heart rate, is more cumbersome, time consuming and expensive. The modified biophysical profile (MBPP) suggested by Nageotte et al combines non stress test (NST) as a short term marker of foetal status and the amniotic fluid index (AFI) as marker of long term placental function is easier to perform and less time consuming than classical biophysical profile.
\end{abstract}

Objectives:

1) To assess the role of modified biophysical profile as a method ofantepartum foetalsurveillance test in predicting perinatal outcome in oligohydramnios cases.

2) To compare the morbidity and mortality with respect to each of the parameters ofmodified biophysical profile, that is NST and AFI individually.

Methods:This study was a prospective clinical study which consisted of 60 pregnant women with oligohydramnios. The patients were evaluated with themodified biophysical profile consisting of NST recording for 20mins, followed byultrasound assessment of amniotic fluid volume, using four quadrant technique.

Results:

- When the Modified biophysical profile is normal, it gives reassurance that thefoetal status is good with good perinatal outcome.

- When the MBPP is abnormal there is increased incidence of perinatalmorbidity as well as mortality.

When considered individually, abnormal AFI was associated with increasedincidence of perinatal morbidity and abnormal NST was associated with increasedincidence of perinatal morbidity as well as perinatal mortality.

Interpretation and conclusion:Modified biophysical profile is an effective method ofantepartum foetalsurveillance test in oligohydramnios in predicting perinatal outcome.

Key words: Modified biophysical profile (MBPP), biophysical profile (BPP), non-stresstest (NST), amniotic fluid index (AFI), foetal heart rate (FHR), amniotic fluidvolume (AFV).

\section{Introduction}

Amniotic fluid plays an important role in foetal health and development.It plays a major role in the development of the foetus. Amniotic fluid allows for proper growth and development of the foetal lung andmusculoskeletal system, has bacteriostatic and anti-inflammatory properties, andaids in thermoregulation, to name but a few of the important functions ${ }^{1}$.It provides a medium in which the foetus can readily move and grow as well. Also, cushions the foetus against the external injuries.

During labour, it helps in even distribution of the pressure of uterine contraction over the foetus, prevents cord compression and helps in cervical dilatation.

The most common intrinsic abnormalities that were encountered clinically were either too much or too little amniotic fluid. Abnormalities of the amniotic fluid volume can interfere directly with the foetal development or may be an indirect sign of underlying disorder.

Normally, amniotic fluid volume reaches $1000 \mathrm{ml}$ by 36 weeks anddecreases thereafter to less than $200 \mathrm{ml}$ at 42 weeks. Diminished fluid is termed oligohydramnios. Oligohydramnios is defined as an AFI of $5 \mathrm{~cm}$ or less at term. $^{2}$

Oligohydramnios can be associated with foetal congenital anomalies and IUGR, it is usually proportional to the degree of IUGR and it indicates placental dysfunction.

Oligohydramnios can also cause asymmetrical foetal growth, contracture of the joints and hypoplasia of foetal lungs by decreasing the lungexpansion due to compression of the foetal abdomen which limits the 
movements of the foetal diaphragm and decreases the flow of the amniotic fluid into and out of thefoetal lung. It is associated with FHR abnormalities, cord compression, poor tolerance of the labour by the foetus, low APGAR scores with a poor perinatal outcome.

From hospital records, it is observed that the average incidence of oligohydramnios is 3 to $4 \%$. Oligohydramnios pregnancies need to be identified so that appropriate surveillance and timely intervention can be employed and thus bring down the rate of perinatal morbidity and mortality.

Antenatal foetal surveillance is directed at identifying foetuses of the high risk pregnancy group such as oligohydramnios. Methods for foetal risk determination have shifted from less specific biochemical methods (e.g. maternal estriol determination) to more specific biophysical testing.

Since the 19th century, foetal assessment consisted of auscultation of foetal heart sounds and subjective recording of foetal movements.In the 20th century, these techniques have been augmented by electronic foetal heart rate monitoring and sonographic evaluation of foetal activity and amniotic fluid volume.

The foetal biophysical profile is one of the most widely accepted tests for the evaluation of foetal well being in such high risk cases. The original biophysical profile was described by Manning et al, which includes study of five variables i.e. breathing movements, foetal tone, foetal body movements, amniotic fluid index and non-stress test. It needs two phase testing byultrasound and external doppler monitor to record foetal heart rate. The complete biophysical profile is more cumbersome, time consuming and is more expensive.

The modified biophysical profile (MBPP) suggested by Nageotte et al combines non stress test (NST) as a short term marker of foetal status and the amniotic fluid index (AFI) as a long term marker of placental function. It is easier to perform and less time consuming than complete biophysical profile. The American College of Obstetricians and Gynaecologists and the American Academy of Pediatrics (2007) have concludedthat the modified biophysical profile test is as predictive of foetal well-being as other approaches to biophysical foetal surveillance. ${ }^{3}$

Hence in this study, Modified BioPhysical Profile is used as a method of antepartum surveillance test in oligohydramnios cases to study its effectiveness inpredicting perinatal outcome.

\title{
II. Materials \& Methods
}

A prospective clinical study was conducted in the department of Obstetrics and Gynaecology at Dr.Pinnamaneni Siddhartha Institute of Medical Sciences\& RF, Chinaoutpalli, which is a teaching hospital. Besides being a teaching hospital, it serves as a main referral hospital for the surrounding district.

\section{Source of data:}

60 pregnant women, $>32$ weeks with oligohydramnios attending the antenatal outpatient clinic or admitted to the wards,during a period of two years from June 2011 to October 2013were considered as the "test group".

\section{Inclusion criteria:}

- Pregnant women with oligohydramnios(AFI $</=5)$.

- Antenatal women between the age group of 18 (teenage) -35ys (advanced maternal age) will be included.

- Primi and multi gravida will be included.

- Patients already started treatment outside will also be included.

- Pregnant women with gestational age $>32$ weeks.

- Singleton pregnancy.

- Non anomalous foetus.

\section{Exclusion criteria:}

- Pregnant women with normal or increased amount of liquor.

- Pregnant women with any risk factor.

- Pregnant women with gestational age $<32$ weeks.

- Multiple pregnancies.

- Pregnancies after assisted reproductivetechnique(ART) methods.

\author{
Parameters That Have Been Taken In To ConsiderationFor Foetal Outcome: \\ Neonatal \\ - APGAR score \\ - Asphyxia \\ - NICU admissions
}


- $\quad$ Birth Weight

\section{Others}

- Meconium stained liquor

Method of collection of data:

into the study.

After taking written and informed consent and fulfilling the inclusion criteria, patients were included

\section{Method of study:}

A detailed history of the pregnant women included in the study was taken andthorough clinical examination including recording of vital parameters, Systemic and Obstetric examination was carried out at booking or admission. Allpreliminary investigations including ultrasound were done.

The patients were evaluated with the modified biophysical profile consistingof NST recording for 20 mins, followed by amniotic fluid index measurement using four quadranttechniques. The test was initiated at 32 weeks of gestation or at the gestational age at which oligohydramnios was identified after 32 weeks. The test was repeated bi-weekly. The patients were treated by modalities of Bed rest, Maternal Hydration and Nitric oxide donors accordingly.

\section{Test results were documented as follows:}

The NST was performed with cardiotocogramin Semi-Fowlers position. Speed of the trace was $1 \mathrm{~cm} / \mathrm{min}$. Foetal heart rate, foetal movements and uterine contractions were recorded. The trace was considered as reactive, if more than 2 foetal movements with acceleration of more than or equal to 15 beats/minute lasting for more than or equal to 15 seconds, with good beat-to-beat variability and no decelerations. If the reactive pattern was not recorded within 20 minutes period, the foetus was stimulated with VAST (foetal acoustic stimulator), or administration of a glucose containing beverage and the test continued for another 20 minutes period. If there is no reactivity in this extended period, the trace was deemed non-reactive.

Real-time ultrasound scanning was performed using a $3.5 \mathrm{MHz}$ sectorprobe and general survey of foetus was done and presentationnoted.The volume of amniotic fluid was measured according to the four quadrant technique described by Phelan et $\mathrm{al}^{59}$. With the patient in supine position, uterus was divided into four equal quadrants by two imaginary lines. The vertical line corresponding to linea alba and a transverse line equidistant from pubic symphysis to the top of the fundus. The transducer was held vertically along the maternal longitudinal axis. An AFI was obtained by summing up the depths of largest vertical pockets, which is cord free in all the four quadrants. An AFI of $>5$ was considered normal and less than or equal to five was considered as oligohydramnios. Patient's management was decided upon gestational age and MBPPresults. The last observation of MBPP before 3days of delivery was compared with outcome of pregnancy.

\section{End points to assess outcome of pregnancy}

- Meconium staining of liquor

- 5 minute Apgar score $<7$ was considered as abnormal.

- Admission to NICU

- Perinatal morbidity

- Perinatal mortality

\section{Interpretation of MBPP and action}

- If both tests were normal -bi weekly foetal surveillance with MBPP.

- If both tests were abnormal - management depends on gestational age. If gestational age $>36$ weeks - Delivery

If gestational age $<36$ weeks - Management is individualized.

- If NST is reactive, but AFI is decreased - evaluate for chronic foetal conditions particularly congenital abnormalities and perform MBPP twice weekly.

- If AFI is normal and NST is non-reactive - NST is repeated.

On repetition If NST reactive - bi weekly foetal surveillance with MBPP.

NST non-reactive -Delivery

\section{Statistical analysis:}

A descriptive statistics i.e. percentages and frequencies were calculated. Chi-square test was used to test the association between the variables. Binary logistic regression was applied to measure the risk associated with modified biophysical profile results. $Z$ test (proportion) was applied to find the significant difference. 


\section{Results And Observations}

The study group consisted of 60 women having pregnancy with oligohydramnios attending the antenatal outpatient clinic or admitted to the wards in the Obstetrics and Gynaecology department of Dr. Pinnamaneni Siddhartha Institute of Medical Sciences\& Research Foundation, Chinaoutpalli,fromJune-2011 to October-2013.

A detailed history was taken and thorough systemic and obstetric examination was done. The women were evaluated with the modified biophysical profile consisting of NST recording for 20mins, followed by amniotic fluid index measurement using four quadrant techniques. The test was initiated at 32 weeks of gestation or at the gestational age at which oligohydramnios was identified after 32 weeks.

The test was repeated bi-weekly.The results and observations recorded in the study were evaluated under the following parameters.

\section{Age distribution:}

It is observed that, out of the 60 women $8(13 \%)$ belong to the age group between $18-20$ years. Majority of the cases i.e.,32 women (53\%) belong to the age group of 21-25years. 14 women (23\%) belong to age group of 26-30 years. 5 women (8\%) were aged between 31-35 years and only one women among the study group was aged $>35$ years.

\section{Distribution of booked and un-booked cases:}

In the present study, majority of the cases (75\%) were booked and $25 \%$ were un-booked.

\section{Gestational age wise distribution of cases:}

When the patients were categorized according to the gestational age in weeks, majority of the patients belonged to the gestational age between $36-37$ weeks (35\%).

\section{Gravida distribution:}

Majority of the cases were primi gravida (50\%), followed by 2 nd gravida(33.33\%). $11.67 \%$ of the cases were 3rd gravida. 4 th gravida constituted $5 \%$.

\section{First AFI results:}

The amniotic fluid index was $4.1-5$ in 36 cases $(60 \%)$ and was in between $3.1-4$ in 16 cases (26.67\%) and between $2-3$ in 8 cases $(13.33 \%)$.

\section{Last AFI Test Results:}

The amniotic fluid index in majority of cases i.e., $27(45 \%)$ increased between $5.1-6$, in 20 cases (13.33\%) increased to $6.1-7$, in 6 cases $(10 \%)$ increased to between $7.1-8$, in 6 cases $(10 \%)$ persisted or decreased to between $4.1-5$ and in one case (1.67\%) remained same.

\section{FirstNST results:}

The first NST test results were reactive in 54 cases $(90 \%)$ andnon-reactive in $6(10 \%)$ cases.

\section{LastNSTresults:}

The last NST test results were reactive in 40 cases $(66.67 \%)$ andnon-reactive in $20(23.33 \%)$ cases.

\section{First MBPP results:}

Among the modified biophysical profiles done in 60 cases, both parameters were abnormal in 6 cases $(10 \%)$, NST was normal and AFI was abnormal in the remaining 54 cases $(90 \%)$.

\section{Last MBPP results:}

Among the modified biophysical profiles done in 60 cases both parameters (NST and AFI) were normal in 37 cases $(61.67 \%)$, both parameters were abnormal in 4 cases $(6.67 \%)$, NST is normal and AFI is abnormal in 3 cases (5\%), AFI is normal and NST is abnormal in 16 cases $(26.67 \%)$.

Thus there is increase in cases with both parameters normal and AFI normal and NST abnormal, there is gross decrease in cases with NST normal and AFI abnormal. This indicates that there is an increase in amniotic fluid index.

\section{Number of MBPP'S performed:}

Majority (35\%) of the cases in the study group had four MBPP tests performed. Twelve MBPP tests were performed in 1 case. 


\section{Last test and delivery interval:}

Majority of the cases $41(68.33 \%)$ delivered within 12 hours of conducting the last test. 8 cases (13.33\%) delivered within 12 to 24 hours, 5 cases $(8.33 \%)$ delivered within 24 to 36 hours, 4 cases $(6.67 \%)$ delivered within 36 to 48 hours and two cases (3.33\%) delivered after 48 hours.

\section{ModeofDelivery:}

Out of 60 cases $28(46.67 \%)$ had vaginal delivery and $32(53.33 \%)$ had caesarean section. Out of the 28 vaginal deliveries $19(31.6 \%)$ were full term and $9(15 \%)$ were preterm. Out of the 32 cases that had caesarean section 22 were full term and 10 were pre term. Out of 22 full terms LSCS 14 (23.33\%) were emergency and 8 $(13.33 \%)$ were elective. All the preterm LSCSswereemergency.

Table 1: Modes of Delivery

\begin{tabular}{|c|l|l|l|}
\hline \multirow{3}{*}{ Vaginal (28) (46.67\%) } & Full term 19 & $31.67 \%$ & \\
\cline { 2 - 4 } & Pre term 9 & $15.00 \%$ & \\
\hline \multirow{3}{*}{ LSCS (32) (53.33\%) } & Full Term 22 & Emergency 14 & $23.33 \%$ \\
\cline { 2 - 4 } & \multirow{3}{*}{ Pre term 10 } & Elective 8 & $13.33 \%$ \\
\cline { 2 - 4 } & & Emergency 10 & $16.67 \%$ \\
\hline
\end{tabular}

Indications for LSCS: Out of the 32 cases who underwent caesarean section majority of them i.e. 20 cases $(62.5 \%)$ hadfoetal distress as the indication for LSCS. Other indications were cephalo pelvic disproportion (9.38\%), previous caesarean pregnancy with scar tenderness in $15.63 \%$, and breech presentation in $12.5 \%$ of cases.

Table 1: Indications For LSCS

\begin{tabular}{|l|r|r|}
\hline \multicolumn{1}{|c|}{ Indication } & \multicolumn{1}{c|}{ Number } & \multicolumn{1}{c|}{$\%$} \\
\hline Foetal Distress & 20 & $62.50 \%$ \\
\hline Cephalo Pelvic Disproportion & 3 & $9.38 \%$ \\
\hline Breech & 4 & $12.50 \%$ \\
\hline Previouos caesarean section & 5 & $15.63 \%$ \\
\hline Total & 32 & \\
\hline
\end{tabular}

\section{Last test result versus mode of delivery:}

Among the modified biophysical profiles done in 60 cases when both parameters (NST and AFI) were normal (37 cases) 11(29.72\%) cases underwent LSCS and 26(70.27\%) cases had vaginal delivery, when both parameters were abnormal all the 4 cases underwent LSCS, when NST is normal and only AFI is abnormal 1 (33.33\%) case underwent LSCS and 2 (66.66\%) had vaginal delivery, when AFI is normal and NST is abnormal all the 16 cases underwentLSCS.

This suggests that the rate of caesarean section is high when either of both parameters were abnormal or when NST is abnormal.

Table 3: Last Test Result vs Mode of Delivery

\begin{tabular}{|l|r|r|}
\hline \multicolumn{1}{|c|}{ MBPP } & Vaginal & \multicolumn{1}{c|}{ LSCS } \\
\hline Both parameters normal (37) & 26 & 11 \\
\hline Both parameters abnormal (4) & 0 & 4 \\
\hline NST normalAFI abnormal (3) & 2 & 1 \\
\hline AFI NormalNST Abnormal (16) & 0 & 16 \\
\hline Total & 28 & 32 \\
\hline
\end{tabular}

Chi-square value is $26.26 . \mathrm{P}$ value $<0.0001$, is significant.

\section{Last NST result versus mode of delivery:}

Of the 60 cases, when NST was considered individually with the mode of delivery, the observations were as follows; when NST was reactive 12 cases had LSCS and 28 cases had vaginal delivery. When NST was non-reactive all the 20 cases underwent LSCS.

This indicates that when NST is abnormal rate of caesarean sections is high.

Chi-square value is 26.25 , $\mathrm{P}$ value $<0.0001$, is significant. 
Last AFI result versus mode of delivery:

Ofthe60 cases, when AFI was considered individually with the mode of delivery, the observations were as follows; when AFI was abnormal $(<=5) 5$ cases had LSCS and 2 cases had vaginal delivery. When AFI was normal $(>5) 27$ cases had LSCS and 26 cases had vaginal delivery.

This indicated that when AFI was abnormal, rate of caesarean sections was high.

Chi-square value is $1.043, \mathrm{P}$ value 0.3072 , is not significant

\section{Last test result versus meconium staining of Liquor:}

Out of 60 cases meconium staining of liquor waspresent in 17 cases. When both parameters (NST and AFI) were normal, out of 37 cases 2 cases hadmeconium stained liquor. When both parameters were abnormal all 4 cases had meconium stained liquor. When NST was normal and AFI was abnormal, 2 cases out of 3 had meconium stained liquor and when AFI was normal and NST was abnormal, 9 cases had meconium stained liquor.

Chi-square value is $28.01, \mathrm{P}$ value $<0.0001$, is significant.

\section{Distribution of weight of the baby:}

Majority of the babies had birth weight between $2.6-3 \mathrm{kgs}$ (41.67\%), followed by $28.33 \%$ of the babies whose birth weight was between 3.1-3.5 kgs, followed by $25 \%$ of the babies whose birth weight was between $1.6-2.5 \mathrm{kgs}$. Those with $<1.5 \mathrm{~kg}$ birth weight constituted $3.33 \%$ and those with birth weight between $3.6-4$ kgs constituted only $1.67 \%$.

\section{Last test result versus APGAR score at five minutes:}

Among the 60 cases included in the study, APGAR score of $<7$ was observed among 10 cases. When both parameters ( NST and AFI) were normal 1 case had APGAR score of $<7$, when both parameters were abnormal 2 cases had APGAR score of $<7$, when NST was normal and AFI was abnormal 2 cases had APGAR score of $<7$ and when AFI was normal and NST was abnormal 5 cases had APGAR score of $<7$.

Table 4: last Test Result vs APGAR Score at Five Minutes

\begin{tabular}{|c|c|c|}
\hline MBPP & APGAR $<7$ & APGAR $>=7$ \\
\hline Both parameters normal (37) & 1 & 36 \\
\hline Both parameters abnormal (4) & 2 & 2 \\
\hline NST normalAFI abnormal (3) & 2 & 1 \\
\hline AFI NormalNST Abnormal (16) & 5 & 11 \\
\hline
\end{tabular}

Chi-square value is $16.24, \mathrm{P}$ value 0.001 is significant.

\section{Perinatal morbidity associated with test results}

When both parameters (NST and AFI) were normal perinatal morbidity was present in $11(29.73 \%)$ cases, when both parameters were abnormal, $3(75 \%)$ of them had perinatal morbidity. When NST was normal and AFI was abnormal perinatal morbidity was present in 2(66.67\%) cases and when AFI was normal and NST was abnormal, $9(56.25 \%)$ of them had perinatal morbidity.

This suggests that whenever both parameters are abnormal or even one of the parameters is abnormal there is increased incidence of perinatal morbidity.

Table 5: Perinatal Morbidity Associated with Test Results

\begin{tabular}{|c|c|c|}
\hline MBPP & Number & $\mathbf{\%}$ \\
\hline Both parameters normal (37) & 11 & $29.73 \%$ \\
\hline Both parameters abnormal (4) & 3 & $75.00 \%$ \\
\hline NST normalAFI abnormal (3) & 2 & $66.67 \%$ \\
\hline AFI NormalNST Abnormal (16) & 9 & $56.25 \%$ \\
\hline
\end{tabular}

Chi-square value is $10.65, \mathrm{P}$ value 0.0138 is significant.

\section{Perinatal mortality associated with test results:}

When both parameters (NST and AFI) were normal perinatal mortality was not present in any of the cases, when both parameters were abnormal,1(25\%) of them had perinatal mortality. When NST was normal and AFI was abnormal perinatal mortality was not present in any of the cases and when AFI was normal and NST was abnormal, 1 of them had perinatal mortality.

This suggests that abnormal MBPP and abnormal NST increased incidence of perinatal mortality. 
Table 62: Perinatal Mortality Associated with Test Results

\begin{tabular}{|l|r|r|}
\hline \multicolumn{1}{|c|}{ MBPP } & Number & \% \\
\hline Both parameters normal (37) & 0 & 0 \\
\hline Both parameters abnormal (4) & 1 & $25 \%$ \\
\hline NST normalAFI abnormal (3) & 0 & 0 \\
\hline AFI NormalNST Abnormal (16) & 1 & $6.25 \%$ \\
\hline
\end{tabular}

Chi-square value is $7.629, \mathrm{P}$ value 0.05 is significant.

\section{Discussion}

One of the major goals of antepartum foetal surveillance is early identificationof the compromised foetus and timely intervention. There were various methods ofantepartum foetal surveillance. The best method is the one, which aims at identifyingthe foetus which is at risk, but still in an uncompromised state and requires immediateintervention. In the present study, the modified biophysical profile (MBPP), which isacombination of two parameters, is used as primary surveillance test for oligohydramnios cases. The two parameters were nonstress test (NST), which is a short term markerof foetal status \& amniotic fluid index (AFI), a long term marker of placental function.

The study group consisted of 60 pregnant women with oligohydramnios. Majority of the patients were primigravida (50\%) and majority of them werein the age group of $21-25 \mathrm{yrs}(53 \%)$.

The surveillance of patients in study group is initiated at $32 \mathrm{wks}$ of gestation,as NST is more reliable after 32 weeks. But majority of the patients in our study hadinitiation of MBPP testing from 36 weeks onwards. This was because of the late referral of patients. In the present study, there were 6 cases where testing was initiated at 32 weeks of gestational age.

The mean gestational age in the present study is 35.9 and 36.9 weeks, which is comparable with mean gestational age of 37.5 weeks in a study by Cassey et al.

The non-reactive non-stress test rate is high in women with AFI $<5 \mathrm{~cm}$. In the present study theoccurrence of non-reactive NST is $33.33 \%$ in oligohydramnios,

There were 322 MBPP tests performed on 60 patients. The number of patients undergone 4 tests constituted $35 \%$. The highest number of tests performed was 12 in one patient. The last test done showed that $61.67 \%$ of the MBPP test results as normal(ie., both NST and AFI were normal), $6.67 \%$ as abnormal((ie., both NST and AFI were abnormal), only NST is abnormal in $26.67 \%$ and only AFI is abnormal $(<5 \mathrm{cms})$ in $5 \%$ cases.

Of the 60 NST's in the last MBPP, $66.66 \%$ were reactive \& $33.33 \%$ were nonreactive. The AFI values were $>5$ in $88.3 \%$ of the cases. Earlier works by Miller et al(1996) and Eden et al (1998) also showed similar results.

The mode of delivery in the study group with respect to last MBPP resultshowed that when MBPP is normal with respect to both parameters (37 cases), theincidence of LSCS and vaginal delivery among these were $11(29.72 \%)$ and $26(70.27 \%)$ respectively.

When the MBPP is abnormal with respect to both parameters ( 4 cases), all the $4(100 \%)$ had LSCS.

When the MBPP is abnormal with respect to $\operatorname{AFI}(3$ cases), 1case $(33.33 \%)$ had LSCS and 2 cases $(66.66 \%)$ had vaginal delivery.

When the MBPP is abnormal with respect to NST(16 cases), all the 16 cases had LSCS.

This shows that the mode of deliveryin most of the cases isvaginal when MBPP is normal and the incidence of LSCS in cases where MBPP isabnormal is increased.

When studied with respect to the last MBPP, when both NST and AFI were abnormal 100\% (all 4 out of 4 cases) has thick meconium. When only NST is abnormal $56 \%$ (9 out of 16) hasthick meconium. When only AFI is abnormal $75 \%$ (2out of 3 ) has thick meconium.

An APGAR score of $<7$ was seen in $16.6 \%$ of the cases in our study group.In the last MBPP when both the parameters were abnormal, $100 \%$ of the cases had APGAR $<7$ whereas when NST was normal and AFI was abnormal, 2(75\%) of the cases had APGAR $<7$. When AFI was normal and NST was abnormal, $5(31.25 \%)$ of the cases had APGAR $<7$.

The mean birth weight in our study is $2.8 \mathrm{kgs}$.

In the present study 21 babies (35\%) were admitted to NICU, which describesperinatal morbidity. This is comparable to earlier study by Compitak K et al, which had $33.3 \%$ of the babies admitted to NICU in his study.

In the last MBPP, when both the parameters were abnormal, $100 \%$ of the cases(4) had NICU admission whereas when only AFI is abnormal, $2(75 \%)$ of the cases had NICU admission. When only NST is abnormal, $6(37.5 \%)$ of the cases had NICU admission.

In our study tachypnoea is the commonest indication for NICU admission. 
In our study, there were $2(3.33 \%)$ perinatal mortalities.In the first case both parameters of MBPPwereabnormal and patient also complained of decreased foetal movements.In the second case NST is abnormal but AFI is normal.

A study by S.K.Patil et al showed a perinatal mortality of 8 out of 650 patients $(1.2 \%)$ and Eden et al had $5.94 \%$ of perinatal mortalities in their study.

From the above discussion, we can conclude that MBPP can be used as amethod of antepartum foetal surveillance test to predict the perinatal outcome in oligohydramnios cases.

\section{Conclusion}

Modified biophysical profile (MBPP) is easier, less time consuming, cost effective and patient compliant test.

- When the Modified biophysical profile is normal, it gives reassurance that the foetal status is good with good perinatal outcome. At the same time, when MBPP is abnormal, it indicates that the foetus may be compromised.

- When the MBPP is abnormal there is increased incidence of perinatalmorbidity as well as mortality. Confirmation with complete biophysical profile can be done when MBPP results were abnormal.

- When considered individually, abnormal AFI was associated with increased incidence of perinatal morbidity and abnormal NST was associated with increased incidence of perinatal morbidity as well as perinatal mortality.

- MBPP can be used as a method of antepartum foetal surveillance test to predict perinatal outcome and provide timely intervention in oligohydramnios pregnancies.

The number of patients included in this study is 60 . To formulate adefinitive protocol, further multicentric studies with larger samples should beconducted.

\section{References}

[1]. Mary B. Munn, MD.Management ofOligohydramniosin Pregnancy.Obstet Gynecol Clin N Am 38 (2011) $387-395$.

[2]. Disorders of amniotic fluid volume,Williams Obstetrics 23rd edition; Chapter 21; p 490-496

[3]. Antepartum assessment, Williams Obstetrics 23rd edition; Chapter 15; p 342.

[4]. Bwerett JM, Sayter SL, Boehm JM.The NST.An evaluation of 1000 patients. Am J Obstet Gynaecol,1981;141-153.

[5]. Vintzileous AM, Campbell WA, Ingardie CJ et al. The foetal BPP and itspredictive value. Obstet Gynecol 1983;62:217.

[6]. Chamberlain PF, Manning FA, Morrison I, Harman CR, Lang CR. The relationship of marginal and decreased amniotic fluid volumes to perinatal outcome. Am J Obstet Gynaecol, 1984; 150: 245-9.

[7]. Crowley P, Herlihy CO, Boylan O. The value of ultrasound measurement ofamniotic fluid volume in the management of prolonged pregnancies.Br J ObstetGynecol, 1984; 91: 444-8.

[8]. Manning F et al. Ultrasound evaluation of amniotic fluid: outcome of pregnancies with severe oligohydramnios. Am J Obstet Gynaecol, 1986 April; 154(4): 895-900.

[9]. Rutherford SK, Phelan JP, Smith CV, Jacob N. The four-quadrant assessment of AFV: An adjunct to antepartum foetal heart rate testing. Obstet Gynecol 1987;70:353-56.

[10]. Phelan JP, AM M-O, Smith CV et al. AFI measurements during pregnancy. J Reprod Med 1987;32:601-4.

[11]. Clark SL, Sahey P, Jolley K. Non-stress testing with acoustic stimulation of AFV assessment in 5973 tests without unexpected foetal deaths. Am J Obstet Gynaecol,1989; vol 148: p-7.

[12]. Brace RA, Wolf EJ. Normal amniotic fluid volume changes throughoutpregnancy. Am J Obstet Gynaecol,1989; vol 161: p- 382388.

[13]. Miller, David A, Robells, Yolanda A, et al. The modified biophysical profile: Antepartum - testing in the 1990's. Am J Obstet Gynaecol : 1996 ; vol 174(3):p-812-817.

[14]. Sood AK, Vibroacoustic stimulation and modified biophysical profile in high riskpregnancy. J Obstet Gynecol India Jan. 2007;57(1):37-41.

[15]. Smith CV. Vibroacoustic stimulation for risk assessment: In: Clinics ofPerinatalogy.1994; 21:797-08.

[16]. Arulkumaran S, Chua S, Obstetrics and Gynecology for Postgraduates. In:RatnamSS, Bhasker Rao K, Arulkumaran S (eds): Hyderabad, Orient Longman1999;1:126-35.

[17]. Maurice M Abitol, Alan G. Monheit et al. Non-stress test and maternal position.Obstet Gynecol Sept 1986; 68:310.

[18]. Freeman R K. The use of oxytocin challenge test for antepartum clinicalevaluation of uteroplacental response. Am J Obstet Gynaec.1975;121:481. 